\title{
Uso do Ecocardiograma Transesofágico na Cardioversão de Fibrilação Atrial
}

\author{
Paulo Roberto Schvartzman, Waldomiro C. Manfroi, Antonio Pinotti \\ Porto Alegre, RS
}

\begin{abstract}
A fibrilação atrial (FA) é a taquiarritmia mais comumente encontrada na prática clínica, cuja prevalência aumenta dramaticamente com a idade ${ }^{1}$. A prevalência de FA no estudo de Framingham é inferior a $1 \%$ na $6^{\circ}$ década de vida, porém pode atingir $9 \%$ em octogenários ${ }^{1}$. A FA é caracterizada por atividade mecânica e elétrica irregular e desorganizada dos átrios, podendo resultar em alteração na capacidade funcional e aumento no risco de insuficiência cardíaca, eventos tromboembólicos e morte ${ }^{1,2}$.

O objetivo do tratamento da FA é manter o ritmo sinusal, controlar a freqüência cardíaca e prevenir o tromboembolismo. O paciente, permanecendo em ritmo sinusal, apresenta benefícios, tais como, eliminação dos sintomas da arritmia, melhora do estado hemodinâmico e diminuição do risco do tromboembolismo ${ }^{1,2}$, o que torna importante, sempre que possível, reverter o ritmo para sinusal, sempre que o paciente se apresentar com FA.
\end{abstract}

\section{Cardioversão}

A cardioversão farmacológica ou elétrica da FA para ritmo sinusal é associada a risco aumentado de tromboembolismo e o uso de anticoagulação diminui esse risco ${ }^{3}$. A cardioversão elétrica está associada a risco de embolização sistêmica que varia de $0,6 \%$ a 5,6\% ${ }^{3,4}$. Tais trombos parecem se originar do átrio esquerdo (AE) e/ou apêndice atrial esquerdo (AAE).

Em 1969, Bjerkuland e Orning ${ }^{4}$ publicaram os resultados de um estudo prospectivo e não randomizado, comparando 437 pacientes com FA que iriam se submeter a cardioversão elétrica para restaurar o ritmo sinusal ${ }^{4}$, com e sem a profilaxia da anticoagulação. A incidência de embolização sistêmica no grupo recebendo tratamento anticoagulante foi de $0,8 \%$, enquanto que no grupo sem anticoagulação foi de 5,3\%. Apesar dos resultados marcantes, esse trabalho recebeu algumas críticas, como ausência de randomização, ausência de descrição dos grupos com FA e questionamento dos níveis de anticoagulação. Entretanto, apesar dessas críticas, as recomendações de anticoagulação para pacientes com FA a serem cardio-

Hospital de Clínicas de Porto Alegre - UFRGS

Correspondência: Paulo Roberto Schvartzman - Rua Marquês de Pombal, 799/ 203 - 90540-001 - Porto Alegre, RS

Recebido para publicação em 7/8/97

Aceito em 2/10/97 vertidos baseiam-se nesse estudo ${ }^{4}$. O American College of Chest Physicians recomenda que pacientes com FA com duração $>48$ h devem receber três semanas de anticoagulação com cumarínicos antes da cardioversão química ou elétrica e quatro semanas após a cardioversão ${ }^{5}$.

\section{Mecanismo de tromboembolismo na fibrilação atrial}

O entendimento do mecanismo envolvido na trombogênese e tromboembolismo em pacientes com FA não é totalmente conhecido. A formação do trombo durante o episódio de FA pode estar relacionado com a tríade de Virchow (lesão no endocárdio, estase no fluxo e estado de hipercoagubilidade). A estase sangüínea e a redução do fluxo parecem ser os fatores mais importantes no desenvolvimento do trombo em pacientes com FA. Evidências de um estado de hipercoagubilidade são escassas, porém investigadores têm sugerido um aumento no fibrinogênio e D-dímero acompanhado de uma diminuição de antitrombina III ${ }^{6}$. A lesão do endocárdio que poderia desencadear o processo trombótico não encontrou, até o momento, fundamentos na literatura.

\section{Mecanismo do tromboembolismo na cardioversão elétrica}

Os pacientes com FA podem apresentar trombos no interior dos átrios e/ou apêndices atriais devido a estase sanguiínea e quando retornam ao ritmo sinusal podem deslocar esses trombos com conseqüente episódio tromboembólico. Porém, em estudo com ecocardiograma com Doppler em pacientes com FA, que iriam ser cardiovertidos, observou-se que o AE demorou até três semanas para contrair com força efetiva ${ }^{7}$. Tal achado forma a base teórica para o regime de anticoagulação por um período de quatro semanas pós-cardioversão, para prevenir a formação de trombo intracavitário, enquanto os átrios retomam a sua função normal.

A maioria dos estudos mostra que os eventos embólicos não ocorrem no momento da cardioversão. O intervalo entre cardioversão e processos tromboembólicos varia de horas a algumas semanas após a cardioversão. Podem existir algumas explicações para tal fato: 1) o retorno da atividade mecânica atrial tarda a ocorrer em relação a atividade elétrica, resultando em expulsão tardia do trombo preexistente ${ }^{8}$; 
2) cardioversão e retorno ao ritmo sinusal resultam em ambiente mais trombogênico devido à lentidão do apêndice atrial em retomar a sua função contrátil, levando a formação de novos trombos e sua expulsão conseqüente ${ }^{8,9}$. Há evidencias para apoiar ambas as teorias.

$\mathrm{O}$ apêndice atrial apresenta um papel importante na formação do trombo por se tratar de uma pequena cavidade. O padrão de contratilidade do AAE já foi estudado através do ecocardiograma com Doppler transesofágico e identificado padrões de fluxo normal em pacientes com ritmo sinusal e fluxos alterados, com fibrilação e flutter atrial e, também, mostrado a grande incidência de trombos no seu interior ${ }^{8}$.

Estudos recentes têm focalizado a questão da etiologia da embolia sistêmica pós-cardioversão. Grimm e col ${ }^{9}$ estudaram 20 pacientes que apresentavam FA e seriam cardiovertidos, realizando ecocardiograma transesofágico (ETE) imediatamente antes e após a cardioversão. A função do AAE foi estudada com Doppler ecocardiograma e a presença ou ausência de trombo e/ou contraste espontâneo foi avaliada. Este estudo apresentou três conclusões principais: 1) a função do AAE retornou imediatamente, embora de forma incompleta, em $80 \%$ dos pacientes após a cardioversão; 2) as velocidades máximas do apêndice atrial antes da cardioversão foram significativamente maiores do que após a cardioversão; 3) o contraste espontâneo no interior do AAE e AE surgiu ou foi intensificada em $35 \%$ dos pacientes. Portanto o AAE e AE apresentaram-se nocauteados imediatamente após a cardioversão, sugerindo que há um novo processo de formação de trombo nessa fase, onde há atividade elétrica nos átrios, mas com a atividade mecânica tardando a se restabelecer.

\section{Implicações clínicas dos pacientes submetidos a cardioversão}

Diversos autores estudaram o papel do ETE em pacientes que realizaram cardioversão de FA, sugerindo que o exame é útil para excluir a presença de trombos em pacientes que não foram adequadamente anticoagulados ou que o exame negativo para trombo poderia reduzir o tempo de anticoagulação.

Moreyra e $\mathrm{col}^{12}$ realizaram uma análise de vários trabalhos com número pequeno de pacientes, comparando três grupos de pacientes com FA: 1) cardioversão em pacientes comETE sem anticoagulação; 2) cardioversão em pacientes anticoagulados pré e pós-cardioversão e sem ETE; 3) cardioversão em pacientes sem anticoagulação e sem ETE. Demonstraram que a incidência de episódios tromboembólicos foi de $2 \%$ nos grupos 1 e 3 ( $\mathrm{p}=0,26$ ), porém a incidência era menor no grupo $2(0,33 \%)$ quando comparado com grupo $1(1,34 \% ; \mathrm{p}=0,04)$. Esses resultados demonstraram que a cardioversão deve sempre ser acompanhada de anticoagulação e que a cardioversão acompanhada do ETE para exclusão de trombos sem anticoagulação apresenta uma incidência elevada de episódios tromboembólicos.

Manning e $\mathrm{col}^{13}$ estudaram com ETE 230 pacientes hospitalizados que apresentavam FA com duração $>48 \mathrm{~h}$ ou desconhecida e que seriam submetidos a cardioversão, estando esses anticoagulados no dia do exame transesofágico e mantidos por quatro semanas após a cardioversão. O estudo demonstrou que $15 \%$ dos pacientes apresentavam trombos intracavitários e a cardioversão foi postergada. A cardioversão obteve sucesso em $54 \%$ com medicação e $46 \%$ com eletricidade. Após a cardioversão com sucesso todos os pacientes foram tratados com cumarínicos por quatro semanas e sem nenhum evento tromboembólico ocorrido, demonstrando que o ETE precedendo a cardioversão na FA é seguro e é uma alternativa para a rotina habitual de anticoagulação por três semanas antes da cardioversão. Deve ser ressaltada a necessidade de heparinização seguida de cumarínico antes e após a cardioversão.

Stoddard e col ${ }^{14}$ também estudaram com ETE pacientes com FA que iriam ser cardiovertidos e também identificaram que cerca de $14 \%$ dos pacientes apresentavam trombo no interior dos átrios e/ou apêndice atrial. Porém estudos demonstram que os processos embólicos ocorrem em cerca de $2 \%$ a $5 \%$ com FA ${ }^{1,2,5,15}$, sugerindo que nem todos trombos preexistentes embolizam durante a cardioversão.

\section{Os átrios nocauteados}

Vários estudos têm demonstrado ausência de trombos no momento da cardioversão através do ETE e quando repetido o exame após a cardioversão observa-se a presença de trombos ${ }^{15-17}$. O mecanismo responsável por esse processo denomina-se nocauteamento atrial e não é totalmente entendido. Inicialmente, acreditava-se que a energia elétrica durante a cardioversão seria a responsável, porém pacientes que retornam ao ritmo sinusal, espontaneamente, também apresentam átrio nocauteado ${ }^{17}$.

Falcone e col ${ }^{18}$ compararam, através do ETE, pacientes que eram submetidos a cardioversão química ou elétrica. Demonstraram que ambos os grupos apresentavam diminuição das velocidades no AAE e aumento na formação de contraste ecocardiográfico espontâneo após cardioversão com conseqüente formação de um processo trombogênico, demonstrando que o nocauteamento atrial não está relacionado com a cardioversão elétrica e sim com processo ainda desconhecido.

\section{Benefícios da cardioversão acompanhada do ecocardiograma transesofágico}

A cardioversão precoce acompanhada de anticoagulação curta e guiada pelo ETE tem algumas vantagens sobre a estratégia habitual. Atualmente tem sido recomendada anticoagulação três a quatro semanas antes e após a cardioversão ${ }^{5}$, o que expõe o paciente a um risco adicional de complicação hemorrágica, pois, duplica sua exposição a anticoagulação sistêmica. A incidência de sangramento parece estar relacionada diretamente com o tempo e com o nível de anticoagulação, sendo muito rara em pacientes anticoagulados por um período $<30$ dias ${ }^{15}$.

A cardioversão precoce também oferece benefícios 
fisiológicos sobre o tratamento convencional. A duração da FA é dos fatores preditores mais importante para dizer quem permanecerá em ritmo sinusal após a cardioversão ${ }^{16}$. Em torno de $60 \%$ dos pacientes hospitalizados por FA estão com esta arritmia por período inferior a um mês e o tratamento tradicional com três a quatro semanas de anticoagulação dobra o período total da arritmia. O tempo necessário para o átrio retornar com sua função mecânica, após a cardioversão, está diretamente relacionada com a duração da FA ${ }^{19}$. Pacientes com FA com duração <2 semanas apresentam recuperação da função mecânica dos átrios em 24h após a cardioversão; aqueles cuja duração do episódio de FA é <6 semanas, apresentam recuperação dessa função em sete dias. Pacientes com a duração da FA > 6 semanas demoram até três semanas para retornar com atividade mecânica nos átrios.

\section{A presença de trombo no ecocardiograma transesofágico}

A conduta ideal com os pacientes em que se encontram trombos atriais com o ETE antes da cardioversão ainda não está definida. Embora o tratamento com anticoagulantes e postergação da cardioversão seja o mais correto, não existe um consenso sobre a manejo desses pacientes. No estudo de Manning e $\mathrm{col}^{13}$, a conduta com os 34 pacientes com trombo atrial incluiu cancelamento da cardioversão, cardioversão após anticoagulação prolongada sem repetir o ETE e cardioversão após documentação da resolução do trombo com o ETE. Três pacientes morreram e, somente, em um deles foi confirmado o episódio tromboembólico.

Klein e $\mathrm{col}^{20}$ sugerem que, ao detectar trombo atrial, seja repetido o ETE em quatro semanas após anticoagulação rigorosa com cumarínicos, mantendo o INR entre 23.É adequado realizar a cardioversão após este período caso o ETE não mais detecte a presença de trombo. Caso o trombo persista após as quatro semanas de anticoagulação, a conduta é mais problemática. Embora a anticoagulação prolongada deve ter permitido a organização e/ou aderência do trombo, o risco de eventos embólicos após a cardioversão não deve ser diferente daqueles pacientes que recebem três semanas de anticoagulação antes da cardioversão eletiva, mas não há dados analisando tal situação. A decisão de realizar cardioversão deve ser individualizada e o riscobenefício da cardioversão nesta situação deve ser avaliado.

\section{Cardioversão com ecocardiograma transesofágico é mais segura do que o tratamento convencional?}

Ao se realizar ETE em paciente que vai ser submetido à cardioversão, detectando a presença de trombo atrial e postergando a cardioversão, estaríamos teoricamente diminuindo o risco de embolia associada ao procedimento. Embora isto pareça ser uma afirmação lógica, não existe embasamento teórico para comprová-la. Os estudos de Manning e $\mathrm{col}^{13} \mathrm{e}$ Stoddard e $\mathrm{col}{ }^{14}$ demonstraram que a abordagem com ETEé segura, porém não sabemos se é mais segura do que o tratamento convencional.

Para responder esta dúvida está sendo realizado um estudo prospectivo, randomizado e multicêntrico, denominado ACUTE (Assessment of Cardioversion Using Transesophageal Echocardiography), em cerca de 3000 pacientes com FA com duração $>48 \mathrm{~h}$, não anticoagulados cronicamente e considerados candidatos à cardioversão. Os pacientes são randomizados para o "braço" do ETE com anticoagulação ou para o tratamento convencional com anticoagulantes. Os pacientes randomizados para o grupo do ETE irão receber heparina e/ou cumarínicos e farão o ecocardiograma e, caso seja excluída a presença de trombos, será realizada a cardioversão e mantida anticoagulação por quatro semanas. Caso se detecte a presença de trombo ou não se visibilize o apêndice atrial, adequadamente, a cardioversão será postergada por quatro semanas e a anticoagulação mantida. Após este período será realizado novo ecocardiograma transesofágico e, caso o trombo não esteja presente, a cardioversão será realizada. Caso o trombo ainda persista, o tratamento será individualizado.

Recentemente, foram publicados os dados preliminares do estudo piloto do ACUTE ${ }^{21}$, tendo sido randomizados 126 pacientes comFA $>2$ dias. Sessenta e dois pacientes foram randomizados para o grupo da cardioversão guiada com ecocardiograma, sendo o ecocardiograma realizado em $90 \%$ dos pacientes e detectado trombo em $13 \%$ desses. Nos pacientes sem trombo foi realizada cardioversão, não ocorrendo nenhum episódio tromboembólico. No grupo com tratamento convencional com cumarínicos foram randomizados 64 pacientes, ocorrendo um episódio de embolia periférica, porém este paciente não se encontrava devidamente anticoagulado, já que apresentava INR 1,8.

A incidência de complicações foi avaliada em ambos os grupos estudados. Observou-se que o sangramento foi discretamente mais freqüente no grupo submetido somente à anticoagulação, porém sem diferença significativa.

Poucos pacientes foram randomizados nesse estudo piloto para tirarmos conclusões a respeito deste tópico, entretanto, alguns pontos devem ser discutidos. Pacientes que necessitavam cardioversão urgente foram excluídos do trabalho; a taxa de sucesso da cardioversão e manutenção de ritmo sinusal entre os dois grupos foi semelhante, sugerindo que as três semanas que antecedem à cardioversão não são de fundamental importância para manutenção do ritmo sinusal, posteriormente.

\section{Conclusões}

O tromboembolismo decorrente da cardioversão é relativamente incomum, suas consequiências podem ser devastadoras e todo cuidado deve ser tomado para evitá-las. Os mecanismos responsáveis pelo tromboembolismo têm sido elucidados e a importância da anticoagulação durante e após a cardioversão está estabelecida. Embora estudos observacionais demonstrem resultados excelentes com ETE na cardioversão, estes devem ser interpretados com certa 
cautela, devido a ausência de grupo controle nos estudos.

Há no momento, duas condutas para o paciente com FA, cujo ritmo pode ser revertido para sinusal: tratamento conservador com anticoagulação ou tratamento curto de anticoagulação acompanhado de ETE. As duas abordagens, pelos dados existentes na literatura até o momento, são seguras e sem diferenças estatísticas em relação às complicações. Portanto, a escolha da conduta depende das preferências do médico assistente e da disponibilidade do ecocardiograma transesofágico.

Em um futuro próximo, espera-se que os resultados finais do estudo ACUTE orientem a conduta mais correta nos pacientes que serão submetidos à cardioversão, melhorando a morbidade desse grupo de pacientes.

\section{Referências}

1. Wolf PA, Abott RD, Kannel WB - Atrial fibrillation as an independent risk factor for stroke: The Framingham Study. Stroke 1991;22: 983-8.

2. Wolf PA, Abott RD, Kannel WB - Atrial fibrillation: A major contributor to stroke in the elderly. The Framingham Study. Arch Intern Med 1987; 147: 1561-4.

3. Lown B, PeLroth MG, Kaidbey S et al - Cardioversion of atrial fibrillation. A report on the treatment of 65 episodes in 50 patients. N Engl J Med 1963; 269: 325-31.

4. Bjerkelund CJ, Orning OM - The efficacy of anticoagulant therapy in preventing embolism related to DC eletrical cardioversion of atrial fibrillation. Am J Cardiol 1969; 23: 208-15.

5 Laupacis A, Albers G, Dunn M et al - Antithrombotic therapy in atrial fibrillation. Third ACCP Conference on Antithrombotic Therapy. Chest 1992; 102(suppl: 4): 26S-33S

6 Gustafsson C, Blomback M, Britton Met al-Coagulation factors and the incresed risk of stroke in nonvalvular atrial fibrillation. Stroke 1990; 21: 47-51.

7. Manning WJ, Leeman DE, Gotch PJ et al - Pulsed Doppler evaluation of atrial mechanical function after eletrical cardioversion of atrial fibrillation and flutter in non-anticoagulated patients. J Am Coll Cardiol 1989; 126: 375-81.

8. Polick C, Taylor D - Assessment of left atrial appendage function by transesophageal echocardiography. Circulation 1991; 84: 223-31.

9. Grimm RA, Stewart WJ, Maloney JD - Impact of eletrical cardioversion for atrial fibrillation on left atrial appendage function and spontaneous echo contrast: caracterization by simultaneous transesophageal echocardiography. J Am Coll Cardiol 1993; 22: 1359-66.

10. Grimm RA, Klein AL, Cohen GI - Tranesophageal echocardiography in patients with atrialarrhytmias undergoing eletrical cardioversion:identification of sources of emboli. PACE 1992; 15: 587.

11. Orsinelli DA, Pearson AC - Usefulness of transesophageal echocardiography to screen for left atrial thrombus before elective cardioversion for atrial fibrillation. Am J Cardiol 1993; 72: 1337-9.
12. Moreyra E, Finkelhor R, Cebul R - Limitations of transesophagealechocardiography in the risk assessment of patients before nonanticoagulated cardioversion from atrial fibrillation and flutter: An analyse of pooled trial. Am Heart J 1995; 129: 71-5.

13. Manning W, Silverman D, Keighley Cet al - Transesophageal echocardiography facilated early cardiovaersion from atrial fibrillation using short term anticoagulation: Final results of a prospective 4.5 year study. J Am Coll Cardiol 1995; 25 : 1354-61.

14. Stoddard MF, Dawkins PR, Prince CR et al - Left atrial appendage thrombus is not uncommun in patients with acute atrial fibrillation and a ecent embolic event. A transesophageal echocardiography study. J Am Coll Cardiol 1995; 25: 452-9.

15. Weinberg DM, Mancini GBJ - Anticoagulation for cardioversion of atrial fibrillation. Am J Cardiol 1989; 63: 745-6.

16. Dethy M, Chassat C, Roy D et al - Doppler echocardiographic predictors of recurrence of atrial fibrillation after cardioversion. Am J Cardiol 1988; 62: 723-6.

17. Grimm RA, Leung DY, Black IW et al - Left atrial appendage stunning after spontaneous conversion of atrial fibrillation demonstrated by transesophageal echocardiography. Am Heart J 1995; 130: 174-6.

18. Falcone R, Morady F, Armstrong W - Transesophageal echocardiographic evaluation of left atrial appendage function and spontaneous contrast formation after chemical or eletrical cardioversion of atrial fibrillation. Am J Cardiol 1996; 78: 435-9.

19. Manning WJ, Silverman DI, Katz SE et al-Impaired left atrial mechanical function after cardioversion: relationship to the duration of atrial fibrillation. J Am Coll Cardiol 1994; 23: 1535-40.

20. Leung D, Grimm R, Klein A - Transesophageal echocardiography-guided approach to cardioversion of atrial fibrillation. Progress Cardiov Dis 1996; 1: 2132.

21. Klein A, Grimm R, Black I - Cardioversion guided by transesophageal echocardiography: The ACUTE Pilot Study. Ann Intern Med 1997; 126: 200-9. 